\title{
Wireless Sensor Node Localization based on LNSM and Hybrid TLBO- Unilateral technique for Outdoor Location
}

\author{
Vivek Kaundal, Paawan Sharma, and Manish Prateek
}

\begin{abstract}
The paper aims at localization of the anchor node (fixed node) by pursuit nodes (movable node) in outdoor location. Two methods are studied for node localization. The first method is based on LNSM (Log Normal Shadowing Model) technique to localize the anchor node and the second method is based on $\mathrm{Hy}$ brid TLBO (Teacher Learning Based Optimization Algorithm)Unilateral technique. In the first approach the ZigBee protocol has been used to localize the node, which uses RSSI (Received Signal Strength Indicator) values in $\mathbf{d B m}$. LNSM technique is implemented in the self-designed hardware node and localization is studied for Outdoor location. The statistical analysis using RMSE (root mean square error) for outdoor location is done and distance error found to be 35 mtrs. The same outdoor location has been used and statistical analysis is done for localization of nodes using Hybrid TLBO-Unilateral technique. The HybridTLBO Unilateral technique significantly localizes anchor node with distance error of $0.7 \mathrm{mtrs}$. The RSSI values obtained are normally distributed and standard deviation in RSSI value is observed as 1.01 for outdoor location. The node becomes $100 \%$ discoverable after using hybrid TLBO- Unilateral technique.
\end{abstract}

Keywords-Log Normal Shadowing Model (LNSM), Teacher Learning Based Optimization (TLBO), trilateration, unilateral, RSSI, ZigBee, Wireless Sensor Network.

\section{INTRODUCTION}

$\mathbf{T}$ HE predominance of wireless sensor networks in the fields of healthcare, disaster management, military applications, emergency services, monitoring traffic, weather forecasting, environmental observations, in IoT (internet of things), biodiversity mapping, intelligent buildings or bridges, preventive monitoring and/or machine supervision and precision agriculture suggest that localization in these fields is inevitable. Localization of a node as adopted by almost all of the schemes is generally classified into two sub categories: (a) Range-based techniques and (b) Range-free techniques.

Range-based techniques are based on estimation or computation distance or angle between two nodes which uses TOA (Time of Arrival), AOA (Angle of Arrival) and a variation of AOA is TDoA (Time Difference of Arrival), Received Signal Strength Indicator (RSSI), distributed acoustic signatures, Statistical signal processing based Direction of Arrival (DOA)[1], to estimate distance between nodes. In ToA and TDoA the nodes must be synchronized in order to receive information and to achieve this synchronization additional hardware is

V. Kaundal \& P. Sharma are with the Department of Electronics, Instrumentation \& Control Engineering, University of Petroleum \& Energy Studies, Dehradun, INDIA (e-mail: vkaundal@ddn.upes.ac.in, paawan.sharma.in@ieee.org).

M. Prateek is with Department of CIT, University of Petroleum \& Energy Studies, Dehradun, INDIA (e-mail: mprateek@ddn.upes.ac.in). required. Angle of arrival (AoA) schemes needs highly directional antennas which demands additional antenna arrays. Though RSSI is very cost effective, its position estimation accuracy is poor. Limitation of hardware and cost of implementation hinders range based schemes usage. On the other hand range-free techniques use information of connectivity and proximity sensing to estimate position of a node. As in most of the practical applications as a coarse estimate is a sufficient, range free scheme acts as a substitute to range based schemes [2]. Popular range free algorithms are Convex Position Estimate CPE as discussed in [3] where the authors have modeled a known network communication which is peerto-peer as a geometric constraint set of node position, and as given in [4], where the authors gives stress on GPS-less and low cost outdoor localization. APIT (Approximate point-intriangulation test) as presented in [5], authors proposes that the defined APIT scheme gives best results when low overhead in node communication is desired and the placement of node is random and also when the radio pattern is irregular. In [6], authors propose a distributed algorithm which localizes a target node which is unaware of its position by finding the centroid of target area. This is done by employing anchor nodes which are aware of their positions.

In the proposed work, the range based technique is used for study purpose and to implement it in finding the probable location of the anchor node. RSSI values have been used as it is a low cost solution with no extra hardware cost. In range based technique the following sensors can be used like ZigBee, Bluetooth, Wi-Fi and RFID etc. Bluetooth is having small range [7] up to $10 \mathrm{mtrs}$ for version 2.0 and up to $100 \mathrm{mtrs}$ for version 4.0 so it is not suitable in our application. RFID again used for small ranges and Wi-Fi consumes high power so the ZigBee is the best option as it consumes very less power, low cost, high reliability [8] and is having inbuilt capability of transmitting RSSI values without using any extra hardware. The RSSI values are used to localize the nodes. Therefore ZigBee suits better in our study. Xbee modules based on ZigBee protocol have been used to get the RSSI values. The localization of anchor node becomes difficult by getting the RSSI values from the Xbee module because of environmental interference, fading etc. The solution to the problem in this paper is given by modeling the wireless channel.

In the current study the RSSI values have been used to localize the anchor node. The fading or fluctuating readings of RSSI is removed by modelling the wireless channel. Two experiments have been studied and conducted in outdoor field. The first experiment is based on calculating LNSM (LogNormal Shadowing Model) parameters. The two nodes, anchor 
as well as pursuit nodes are designed using Xbee transceiver module in it. The wireless channel is modeled using LNSM technique [9]. The anchor node is set in outdoor location and pursuit node is set to localize the anchor node using modeled wireless channel. The probable distance is estimated in between the nodes based on RSSI values received and same is converted into distance using LNSM. Using LNSM, probable distance can be estimated. In second experiment hybrid TLBO- Unilateral technique has been proposed to localize the anchor node. TLBO [10] is Teaching learning Based Algorithm and unilateral technique [11] is the improved trilateration technique. In hybrid TLBO- unilateral technique the pursuit node is subjected to localize the anchor node. TLBO is used because it is capable of learning the tasks, easy and flexible to use. Unlike other optimization algorithms it will not use specific parameters to get tuned. Anchor node becomes fully discoverable for outdoor location. So, the main contribution to this paper is measuring RSSI values to get the estimated distance with proper modeled wireless channel for outdoor locations. The paper also calculates the standard deviation and distance estimation like parameters to localize the anchor node. In later the study will be on TLBO algorithm, unilateral technique and finds how the hybrid TLBO- unilateral will help to localize the anchor node. The RSSI values from modeled wireless channel have been measured in self-designed hardware design and offline training is done in SCILAB virtual environment. The results concluded the path taken by pursuit node to search the anchor node. The statistical analysis is done for the RSSI values obtained and it is proved that the values obtained is normally distributed in outdoor location after implementing hybrid TLBO Unilateral technique.

\section{RELATED WORK}

Study of sensor node localization is very important in the field of wireless sensor networks. Many studies have been conducted for localization of wireless sensor node. Authors in [12] presented brief survey on ingrain challenges and main issues encountered by few existing algorithms of localization techniques, which are assisted by mobile anchors in WSN. In [13], authors discussed Approximate Point in Triangulation (APIT) and showed that it performs best when node placement is random, radio pattern is irregular and when a low communication overhead is desired. Authors demonstrated that accuracy of range-free schemes is sufficient for various applications in wireless sensor networks. But there is a slight degradation in system performance. Anzai D and S hara in [14] discussed RSSI (Received Signal Strength Indicator) localization algorithm based on maximum a posterior (MAP) along with estimation of channel parameters. This method takes into account the knowledge of a priori location information of target. The experimental results along with theoretical analysis of the proposed method prove that the proposed estimation method is superior to the available conventional method by $30 \%-40 \%$ in terms of accuracy of location estimation. The authors in [15] discussed various localization techniques used in coal mines and proposed use of Ultra-Wide Band (UWB) and Time of Arrival(TOA) based localization technique to be used in coal mine is presented. In this work, Matlab simulation of proposed technique is performed indicating better accuracy than RSSI-based ZigBee system used for localization in coal mine. Authors in [16] presented a hybrid localization technique called clustering technique for indoor application comprising of two techniques viz., Angle of Arrival (AoA) and Round-trip time (RTT). Advantage of using the latter is that it avoids the time synchronization between base stations. As the method is proposed for indoor applications the authors has considered the reflections caused by floor and ceiling while showing the robustness of accuracy achieved in both 2D and 3D environments in their results. Also the given method does not need any prior knowledge so as to correct any errors which are caused by multipath propagation ray tracing. The authors in [17] proposed a two-step algorithm for position estimation of target. First step is to estimate parameters like time of arrival of signal from target and its strength. This is followed by statistical or geometric approaches for analysis. Instead of considering multiple observations of signals, authors have only considered a single observation of signals. Authors have investigated various positioning algorithms and discussed their theoretical limitations. Authors in [18] investigated determination of distance between a mobile and an anchor node by using two approaches. First method was based on channel propagation known as LNSM and the second method was based on PSO ANN (particle swarm optimization- artificial neural networks). LNSM was used to estimate the distance and PSO-ANN was used to increase accuracy of distance estimation without additional components. As per the comparative study between various algorithms the proposed hybrid method based on PSOANN showed subsequent improvement in average localization by minimizing the distance error. As the hybrid method of PSO-ANN performed better than similar type of systems, authors have confirmed the applicability in both indoor and outdoor applications. As per authors in [19] main disadvantage of using RSSI based localization is its interference in real time environments and its instability. Therefore authors presented evaluation of Heuristic Environmental Consideration over Positioning System (HECOPS) approach which is more efficient. The work in the paper describes that when the average of last $\mathrm{n}$ estimates are used instead of using only RSSI value to measure distance among nodes, there is $100 \%$ improvement in node position estimation. Authors in [20] discussed their experiences in implementing an anchor free distributed localization (AFL) for real time applications, and proposed improvements. In [21] authors propose an indoor location system based on ZigBee (Zig BEACON) which can be used for Ambient Light Intelligence (AmI) environments. AmI computing refers to environments in electronics which is sensitive and responds to peoples presence. Results shown by the authors in this study prove that ZigBEACON technique significantly improves the accuracy of location in indoor environments. Algorithm proposed in [22] determines the position of object which will be in between a set of calibrated points. This algorithm is based on Bayesian probability and the measured distance between a set of points calibrated. Results of the proposed algorithm gives an accuracy of 1.5 meter which is quite better than the other RSSI based systems. In [23] there is evaluation of few RSSI based localization techniques. The four algorithms proposed and paper have shown better results than RFID tags in terms of accuracy of localization for 
real time live jobsites. In [24] an overview of measurement techniques in terms of localization in sensor networks and one-hop localization algorithms is presented in this paper along with a detailed investigation into multi-hop distance \& connectivity based algorithms. This paper also discusses open research problems in distance based algorithms along with possible set of approaches are provided. There is an exhaustive amount of research work performed by the authors in this article on localization and its measurement techniques in WSN. Along with addressing open research problems authors have also discussed possible solutions that can be applied to these problems, although few of the problem has been addressed and solved till date by the other researchers. Authors in [25] addressed localization of source by using decentralized method of analyzing spatially separated sensor signals which are Time Difference of Arrival (TDOA) based. Due to constraints in bandwidth and power of centralized methods authors in this letter have proposed a decentralized method of localization. Results here prove that the proposed decentralized method gives the same estimate as that of centralized method at a very less cost. The sensor pairing method adopted by the authors requires less bandwidth and also consumes less power than that of centralized methods. Authors of [26] presented a generalized classification of localization algorithms which is divided into two types, range-based and range-free. However difficulty is to classify hybrid solutions into the same range-based and range-free. This paper simplifies this difficulty by dividing the mentioned schemes into two types: Fully-range based, fully range-free and hybrid range-based and hybrid range-free. The comparative study in this paper is mainly on three parameters viz., Network Assumptions, Localization Process and Design Goals. Sensor nodes in [27] discover their position or location by receiving signals from two or three fixed transmitters which are termed as beacons. Position estimation is done by the angle of arrival method which is simple and economical. As each node estimates its own position the density or number of nodes does not affect the system performance. Also as angle of arrival is used for estimation the geographic area of network need not be considered. Major cost of the system is only on the antenna cost for beacon. Simulation results in [18] shows that the error in position estimation is as low as $5 \%$. Stability is a problem of concern in this method for future work has been suggested by the authors. Maximum of 32 nodes were considered which includes 4 anchor nodes also. This article [28] which also has advanced in time present location estimation technique for Ad-hoc sensor networks using distributed iterative algorithms which look quite unique. Using the testbed prototype authors were able to achieve an accuracy of a few $\mathrm{cm}$. Simulation is used to study the performance and scalability. It is shown that TOA (Time-of-Arrival) is a good parameter for fine-grained location estimate. Whereas RF signal strength which is now called as RSSI is not suitable for pin-point localization. Each localization technique presented above in the survey needs some accuracy to localize the node. Many researchers have tried to reduce the localization error and have shown the improvement in terms of error distance in the result obtained either by simulation or by practical approach but still the results are not satisfactory. Therefore a study of LNSM is important to derive a hybrid TLBO-
Unilateral Algorithm that was implemented in SCILAB and hardware node designed to achieve the desirable results. The nodes become fully discoverable after using the hybrid TLBOunilateral algorithm.

\section{Channel Model}

The transmission of message signal through wireless channel is highly affected by environment especially if it is an outdoor location. Therefore it is highly recommended to model the wireless channel. The propagation model like free space model, two ray model and LNSM (Log- Normal Shadowing Model) has been used for the modelling of wireless channel. LNSM is always preferable because one can set the environmental parameters. The LNSM is explained below in the subsection along with the environmental setting of the outdoor environment.

\section{A. LNSM (Log- Normal Shadowing Model)}

The wireless signals to be transmitted are very much affected by impairments such as reflection, diffraction and scattering. So the wireless channel models are designed by analytical as well as empirical methods. In the following wireless channel modelling analytical method has been taken into consideration in which the channel is designed by taking measured data. In empirical method one has to do the curve fitting. In the below mentioned experiments, we have placed the node in outdoor environment and the signal strength has been observed for $1 \mathrm{mtrs}$ distance. $-40 \mathrm{dBm}$ is the standard signal strength of Xbee [8] at $1 \mathrm{~m}$. And accordingly the wireless channel model has been designed.

The several trials have been made in between anchor node and pursuit node from different angle and non-line of sight. The pursuit node is subjected to move in $45^{\circ}$ angle from the anchor node and move to maximum of $100 \mathrm{mtrs}$. The area is almost flat from anchor to pursuit node when pursuit node is moving. This will give different signal strengths and different points. It gives the different channel conditions on the area. The nodes designed are equipped with Xbee which is having RSSI pin to get the signal strength in between two nodes. The distance in between the node can be determine by LNSM [29] by

$$
P_{L(d)} \text { in } d B m=P_{L\left(d_{o}\right)}+10 n \log _{10}\left(d / d_{o}\right)+X_{\sigma}
$$

where $P_{L(d)} \mathrm{dBm}=$ Path loss in mtrs at distance $\mathrm{d}, P_{L\left(d_{0}\right)}$ is path loss in mtrs at distance $d_{o}$ and is a reference distance taken as $1 \mathrm{mtrs}$ with $-40 \mathrm{dBm}$ RSSI values. $n$ is path loss exponent. Path loss exponent is obtained from the analytical method. $X_{\sigma}$ is known as zero mean Gaussian random variable (dB) with standard deviation $\sigma$. The values of path loss exponent $(n)$ for different environmental conditions including the value of $n$ for outdoor location chosen in present study are shown in Table I.

\section{System ARChitecture}

The proposed system is based upon wireless Ad-Hoc Network. The network is equipped with anchor (fixed) node and Pursuit (movable) node. As seen in Fig.1 the anchor node has XBee module- series 2 based on ZigBee protocol. The 
TABLE I

PATH LOSS EXPONENT FOR DIFFERENT ENVIRONMENTS

\begin{tabular}{ccc}
\hline \hline S.No. & Path Loss Exponent (n) & Environment \\
\hline 1. & 2.0 & Free Space \\
2. & $1.6-1.8$ & Inside building (LOS) \\
3. & 1.8 & Supermarket Store \\
4. & 2.09 & Conference room with table chair \\
5. & 2.2 & Factory \\
6. & $2-3$ & Inside factory (no LOS) \\
7. & 2.8 & Indoor residential area \\
8. & 2.4 & Outdoor environment (in current study)
\end{tabular}

hardware node for the same is shown in Fig.2. Xbee Module is capable to make a wireless mesh network in outdoor location. The node is powered by $2600 \mathrm{mAh}$ power bank and it can be extended as per the node's life requirements. The node is able to calculate the local and remote RSSI values in $\mathrm{dBm}$. The anchor node as well as pursuit node is also equipped with SD card module that will store the RSSI values. The same RSSI values are stored in main data center as shown in Fig.1. In the designed node A to B type cable is used to provide battery. For making the node more user friendly a separate nut connector is also given so that one can attach AA batteries if needed. The provision of $+3.3 \mathrm{~V}$ and $5 \mathrm{~V}$ outlets is given to run other devices in the node. Xbee module needs $+3.3 \mathrm{~V}$ while microcontroller and other devices like SD card module, display needs $+5 \mathrm{~V}$. The node is having SPI interface and serial interface via female connectors. For display 20x4 LCD is used. For the same port given one can attach $16 \times 2$ also and the displays backlight is adjusted via preset. Node is provided with ADC interface too if needed for ADC operation. The wireless channel is modeled and equivalent firmware in embedded $\mathrm{C}$ is used in hardware node designed to localize the anchor node. The two nodes start sharing the local as well as remote RSSI values in modeled wireless channel whenever they come in contact to each other.

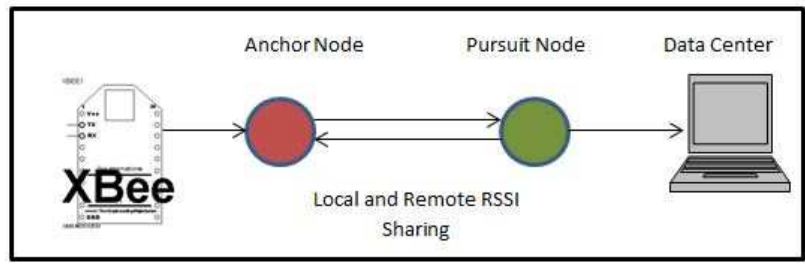

Fig. 1. System Architecture

\section{METHODOLOGY}

The two experiments are performed for measuring RSSI values in outdoor location. The first experiments conducted based on LNSM and second experiment is based on Hybrid TLBOUnilateral technique for the outdoor location (shown in Fig.3). The experiments are performed to find the estimated location of anchor node by measuring the RSSI values. The pursuit node can be located either by classical LNSM technique or by hybrid TLBO- unilateral technique. The outdoor location

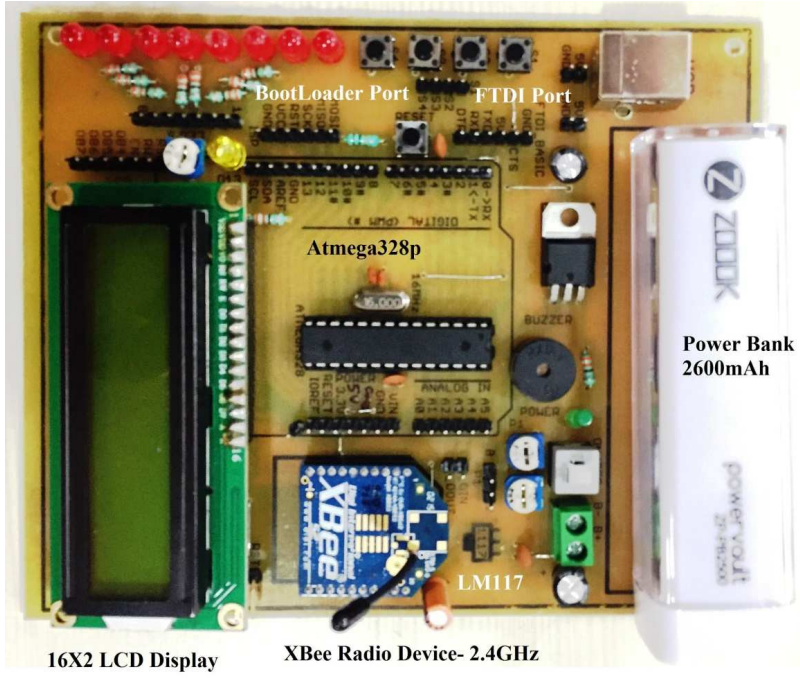

Fig. 2. Hardware Node

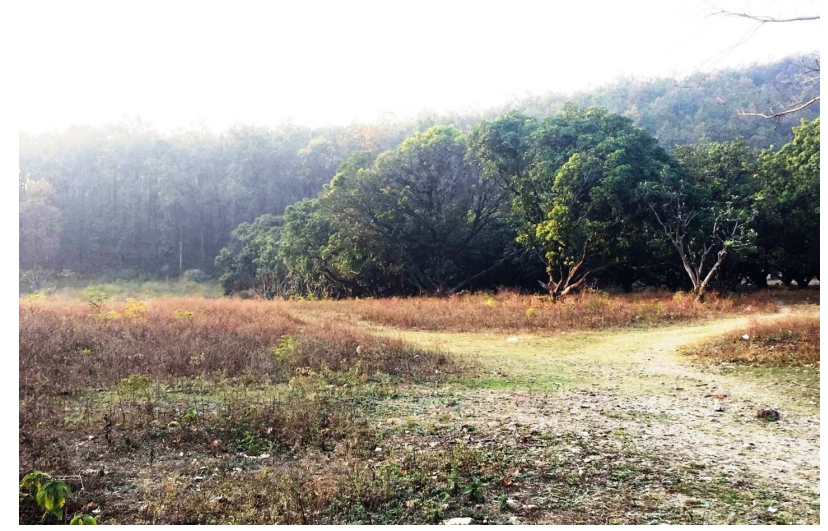

Fig. 3. Outdoor location

in Fig. 3 is 200 x100 mtrs heavy dense forest. Several trials have been made to search anchor node. The nodes are placed $4 \mathrm{mtr}$ above the ground level. The pursuit node is subjected to move around the anchor node but the pursuit node is not aware of the anchor nodes exact location. The sub-sections below show the study of LNSM, TLBO, Unilateral technique and hybrid TLBO- unilateral technique in outdoor location, and how the technique helps in anchor node localization.

\section{A. LNSM Technique}

In first experiment LNSM has been used. The wireless channel is modeled using LNSM as discussed in subsection A of section III. The equivalent firmware is developed in embedded $\mathrm{C}$ and used in self-designed hardware nodes.

\section{B. TLBO (Teacher learning based optimization)}

TLBO is teacher learner based optimization algorithm. It is selected because it does not require algorithm specific parameters unlike GA that uses mutation probability, crossover probability as well as selection operator[10], ABC algorithm requires number of bees and limits, PSO requires inertial 
weight as well as social and cognitive parameters. Likewise other algorithms ES, EP, DE, BFO, AIA, SFL etc. that requires tuning of algorithm specific parameters and if the tuning is not proper then it will increase the computational efforts of the system and become difficult to implement. So the existence of TLBO algorithm for optimization comes into picture. It does not require any algorithm specific parameters. TLBO consist of teacher and learner phase. The equations 2,3,4,5,6,7,8 explained the TLBO in detail [10].

During Teachers phase, for iteration $\mathrm{i}$, there are $\mathrm{m}$ number of design variables and $\mathrm{n}$ numbers of learners of population size, $\mathrm{k}=1,2, . . \mathrm{n}$. and $M_{j, i}$ is the mean result of learners in a subject $\mathrm{j}=1,2, \mathrm{~m}$. The best result overall is $X_{\text {total-kbest, } i}$ is considered as a best learner kbest. Then the best learner in the algorithm is treated a teacher. So the final result is expressed as

$$
\text { Difference } \text { Mean }_{j, k, i}=r_{i}\left(X_{j, k b e s t, i}-T_{F} M_{j, i}\right)
$$

Here $X_{j, k b e s t, i}$ is the result of the best learner in $\mathrm{j}$ subject. $r_{i}$ is the random number. $T_{F}$ is the teaching factor and can be decided by equation (3)

$$
T_{F}=\operatorname{round}[1+\operatorname{rand}(0,1)\{2-1\}]
$$

Based on the solution of Difference Mean $_{j, k, i}$ the teacher phase update the existing solution with equation (4)

$$
X_{j, k, i}^{\prime}=X_{j, k, i}+\text { Difference Mean } n_{j, k, i}
$$

Here $X_{j, k, i}^{\prime}$ is the update value.

During learner phase, learners interact with each other to gather more knowledge.

Here $X_{\text {total-firstvalue }, i}^{\prime} \neq \quad X_{\text {total-Secondvalue }, i}^{\prime}$ where $X_{\text {total-firstvalue }, i}^{\prime}$ and $X_{\text {total-Secondvalue, } i}^{\prime}$ are the updated values of $X_{\text {total-firstvalue, } i}$ and $X_{\text {total-secondvalue, } i}$ in the teacher phase. Both equations 5 and 6 are for minimization problems and for maximization equations 7 and 8 come into existence.

$$
\begin{aligned}
& X_{\text {total-firstvalue }, i}^{\prime \prime}=X_{\text {total-firstvalue }, i}^{\prime}+ \\
& \quad r_{i}\left(X_{\text {total-firstvalue }, i}^{\prime}-X_{\text {total-Secondvalue }, i}^{\prime}\right)
\end{aligned}
$$

if $X_{\text {total-firstvalue }, i}^{\prime}<X_{\text {total-Secondvalue }, i}^{\prime}$

$$
\begin{aligned}
& X_{\text {total-firstvalue }, i}^{\prime \prime}=X_{\text {total-firstvalue }, i}^{\prime}+ \\
& \quad r_{i}\left(X_{\text {total-secondvalue }, i}^{\prime}-X_{\text {total-firstvalue }, i}^{\prime}\right)
\end{aligned}
$$

if $X_{\text {total-firstvalue }, i}^{\prime}>X_{\text {total-Secondvalue }, i}^{\prime}$

$$
\begin{aligned}
& X_{\text {total-firstvalue }, i}^{\prime \prime}=X_{\text {total-firstvalue }, i}^{\prime}+ \\
& \quad r_{i}\left(X_{\text {total-firstvalue }, i}^{\prime}-X_{\text {total-Secondvalue }, i}^{\prime}\right)
\end{aligned}
$$

if $X_{\text {total-secondvalue }, i}^{\prime}<X_{\text {total-firstvalue }, i}^{\prime}$

$$
\begin{aligned}
& X_{\text {total-firstvalue }, i}^{\prime \prime}=X_{\text {total-firstvalue }, i}^{\prime}+ \\
& \quad r_{i}\left(X_{\text {total-secondvalue }, i}^{\prime}-X_{\text {total-firstvalue }, i}^{\prime}\right)
\end{aligned}
$$

if $X_{\text {total-firstvalue }, i}^{\prime}<X_{\text {total-Secondvalue }, i}^{\prime}$

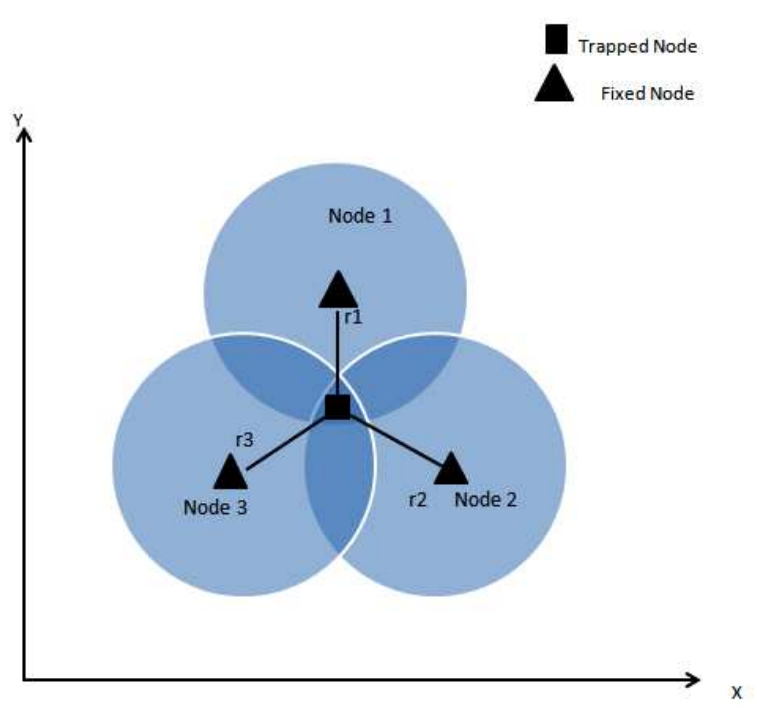

Fig. 4. Trilateration Algorithm

\section{Hybrid TLBO- Unilateral Algorithm}

The most widely proposed and simple method to locate the trapped person is trilateration algorithm (Fig.4) in which four different nodes are present, out of which three become fixed and one moving node is the trapped node. Fairly assuming that all nodes have omnidirectional antenna, each one of fixed node can calculate the Euclidian distance in between. Similarly the unilateral algorithm as shown in Fig.6 is based on searching the anchor (target) node by VPM (Vector Parameter based mapping) [11]. The unilateral technique is improved trilateration technique in terms of number of nodes required to search the anchor. The node will search for RSSI value and once the node will get the RSSI value it will calculated the probable distance. The pursuit node will keep on moving to the target till it will get $-40 \mathrm{dBm}$ RSSI value. Refer Fig.5. In this paper the Unilateral and TLBO will get combine to make a hybrid TLBO- Unilateral Algorithm. In the proposed algorithm pursuit node first get the RSSI values of the anchor node in the nearby areas and teaches the pursuit node to target the anchor node. After the first movement of the pursuit node it will become the learner and start learning the path to follow the maximum value of RSSI till it reaches to $40 \mathrm{dBm}$ RSSI value. The hybrid TLBO-Unilateral technique is shown in Table II. The offline training is simulated in SCILAB virtual environment. The error estimation is based on RMSE (Root mean square error). The probability based localization technique is explained below and function used in below equation is RMSE.

Let us the estimated distance is $d_{E}$, with probability function is $P_{d E i}$ for all $i^{t h}$ node and actual distance is $d_{A}$, with probability function of $P_{d A i}$. Probability theory states that $P_{T i}\left(P_{d E}\right)=P\left(P_{d A i} \mid P_{d E i}\right), P_{T i}$ is the deployment probability field function for anchor node (to be pursued). Considering RSSI value, the probable estimated distance.

$\mathrm{P}_{T i}\left(\mathrm{P}_{d E}\right)=\frac{1}{2 \pi \sigma_{d E}} \exp \left[-\left(\frac{1}{2}\right)\left[\frac{(d a-d e)^{2}}{\sigma_{d E}}\right]\right]$

$\sigma_{d E}=R S S I X P$ is the standard deviation. 


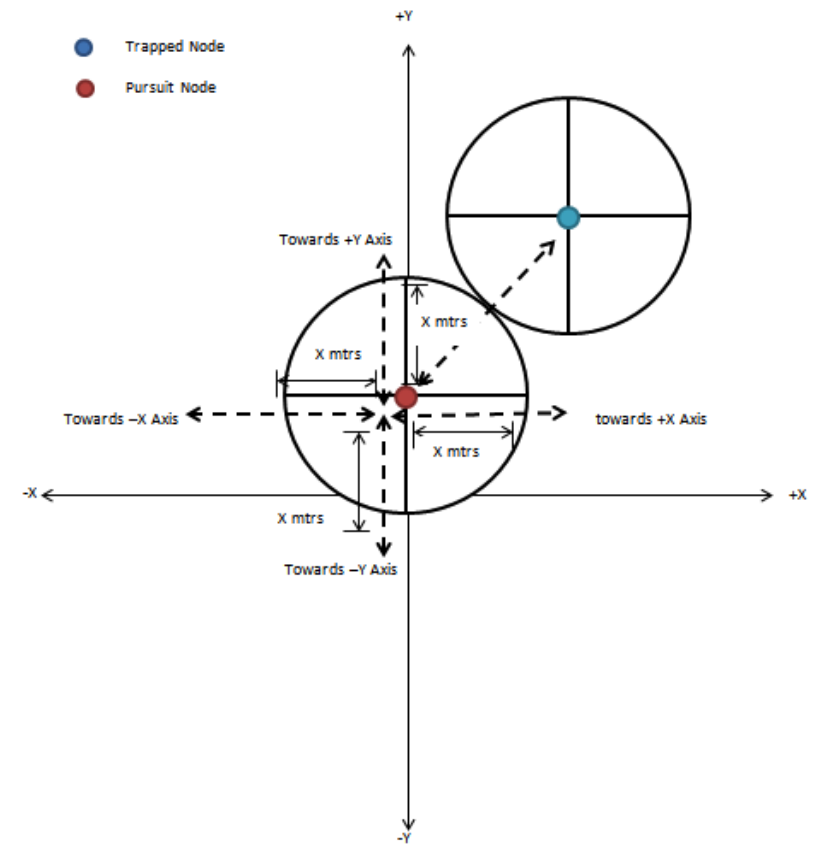

Fig. 5. Unilateral Algorithm

$\mathrm{P}$ is the error factor of the device generally considered as 0.1 . If the anchor node ( $i^{\text {th }}$ node) receives the packet from pursuit node (jth node) the probability function will be defined as

$$
P_{(i, j)}\left(P_{d E i}\right)=P\left(d_{\text {noise }(i j)} \mid P_{d E i}, \widehat{P_{d E j}}\right)
$$

$d_{\text {noise }(i j)}$ is measurement of distance using noisy RSSI value. The RSSI values are Gaussian distributed so the probability function becomes

$$
\begin{gathered}
\mathrm{P}\left(\mathrm{d}_{\text {noise }(i j)} \mid \mathrm{P}_{d E i}, \widehat{P_{d E j}}\right)==P\left(d_{\text {noise }(i j)} \mid \delta\left(P_{d E i}, \widehat{P_{d E j}}\right)\right. \\
P\left(d_{\text {noise }(i j)} \mid P_{d E i}, \widehat{P_{d E j}}\right)== \\
\frac{1}{2 \pi d_{\text {noise }} r} \exp \frac{\left(\delta\left(P_{d E i}, \widehat{P_{d E j}}\right)-d_{\text {noise }(i j)}\right)^{2}}{2\left(d_{\text {noise }(i j)}\right)^{2}}
\end{gathered}
$$

Here $\mathrm{r}=$ range error factor taken as 0.1 . Combining equation 9 and 10

$$
F=\frac{\left(d A_{i}-d_{E i}\right)^{2}}{\sigma^{2} d_{E}}+\sum_{j \in J i} \frac{\left(\delta\left(P_{d A i}, \widehat{P_{d i j}}\right)-d_{n o i s e(i j)}\right)^{2}}{\left(d_{\text {noise.r }}\right)^{2}}
$$

The equation 11 is used in Hybrid TLBO-Unilateral technique to obtain the distance error.

\section{RESULTS \& DISCUSSIONS}

This section is LNSM results and Teaching Learning Optimization Algorithm results have been discussed.

\section{A. LNSM Parameters}

Then LNSM parameters obtained are included in the algorithm designed and used to find the distance and RSSI values. The RSSI values are obtained in outdoor locations as mentioned in above section. 500 Samples of RSSI are
TABLE II

ALGORITHM HYBRID TLBO- UNILATERAL TECHNIQUE

measured for outdoor locations. Each sample is of 50 bytes. The RSSI data measured is shown in Fig.6. The Deviation in outdoor environment is from $-64 \mathrm{dBm}$ to $-68 \mathrm{dBm}$. The deviation is because of the environmental parameters like fading of channel, interferences from trees, building etc. The wireless channel is modeled using LNSM and obtained RSSI by node should follow the normality test. The experiments follows Shapiro-Wilk [29]\& Jarque-Bera test [30]for normality. Based on the RSSI values measured for different locations, the parameters of LNSM can be measured. The regression line can be obtained as shown in Figure 6 .

$$
Y_{\text {outdoor }}=-57.848 X_{\text {outdoor }}+506.8
$$

Note: $Y_{\text {outdoor }}$ is average RSSI and $X_{\text {outdoor }}$ is logarithmic scale of distance in meters.

So we can write the above equations as

$$
R S S I_{\text {outdoor }}=-57.848 \log \left(d / d_{o}\right)_{\text {outdoor }}+506.8
$$

Comparing these equations with LNSM theoretical equation of

$\mathrm{P}_{L(d)} \mathrm{dBm}=\mathrm{P}_{L(d 0)}+10 \mathrm{n} \log 10\left(\mathrm{~d} / \mathrm{d}_{0}\right)+\mathrm{X}_{\sigma}$

We can calculate the path loss exponent and standard deviation as

$10 n=57.848$ and $P_{t}(d B m)-P_{L 0}+X=-506.8($ Outdoor $)$

Therefore with these equations we can find the LNSM parameters. And further the distance can be measured with the following equations:

$$
d=d_{o} 10^{(-(R S S I+506.8) / 57.848)} \text { (in mtrs) Outdoor }
$$

The equation 15 is used to calculate the estimated distance by putting in equation of RMSE (Root Mean Square Error).

$$
R M S E=\sqrt{\left(\frac{1}{n} \sum_{i=1}^{n}(\text { Error })^{2}\right)}
$$

Error= Actual Distance $\left(d_{A}\right)$ - Estimated Distance $\left(d_{E}\right)$.

The RMSE is observed as $35 \mathrm{mtrs}$ in outdoor locations for 20 particles. Also the anchor as well as pursuit node are not normally distributed. In Jarque-Bera normality test Ho is the hypothesis to be assumed to prove the normality. In Jarque Bera test $\mathrm{W}$ is the critical value for the acceptance 


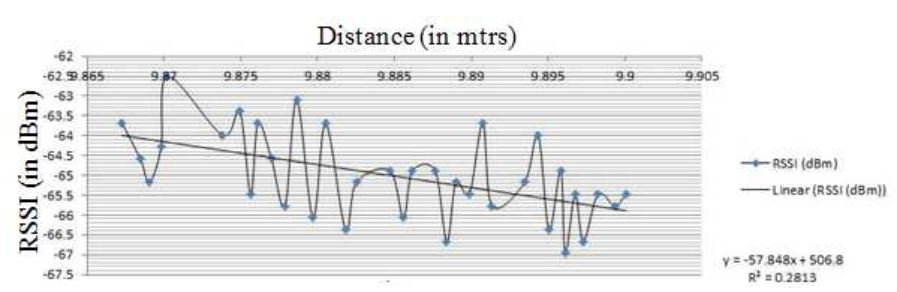

Fig. 6. Measured data in Outdoor Location

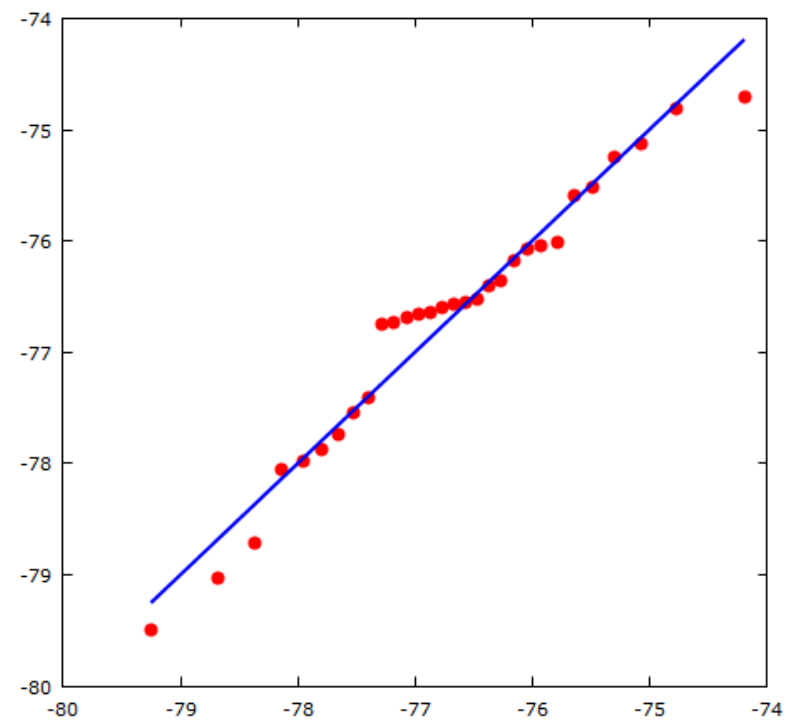

Fig. 7. Plot for Outdoor Location for Normality Check using LNSM

of hypothesis and $\mathrm{W}$ is the critical value. The confidence levels mentioned $90 \%, 95 \%, 97.5 \%, 99 \%, 99.9 \%$ corresponds to $4.61,5.99,7.38,9.21$, and 13.82 respectively. And same for Shapiro- Wilk normality test the critical value $\mathrm{W}$ the confidence level for $90 \%, 95 \%, 98 \%$, and $99.9 \%$ corresponds to the $0.9555,0.947,0.938$, and 0.93 respectively. The RSSI data obtained by modelling of wireless channel using LNSM the Ho for Jarque-Bera and Shapiro-Wilk is not much acceptable at $99 \%$ confidence level. This shows the data is not normally distributed. The Q-Q plot shows the normality behavior of nodes in outdoor location shown in Fig.7. The results are not satisfactory by just modeling of the wireless channel through LNSM. The performances of the nodes in outdoor location are shown in terms of throughput also in Fig.8. It has been observed that the output throughput is not satisfactory, that motivates and leads to the use of Hybrid TLBO- Unilateral technique.

\section{B. Hybrid TLBO Unilateral Technique Results}

In hybrid TLBO- Unilateral technique the wireless channel is modeled using equation 11. And again the normality tests Jarque-Bera and Shapiro Wilk have been studied. Using Hybrid technique $\mathrm{H} 0$ accepted for both test at $99.9 \%$ confidence level shows the normally distribution of the data. As stated in Table III the parameter calculated after obtained RSSI values. The standard deviation is 1.01 and the RMSE calculated using equation 11 is obtained about 0.7 mtrs for 8 search particle. The results in Fig.9 shows the normality behavior of the

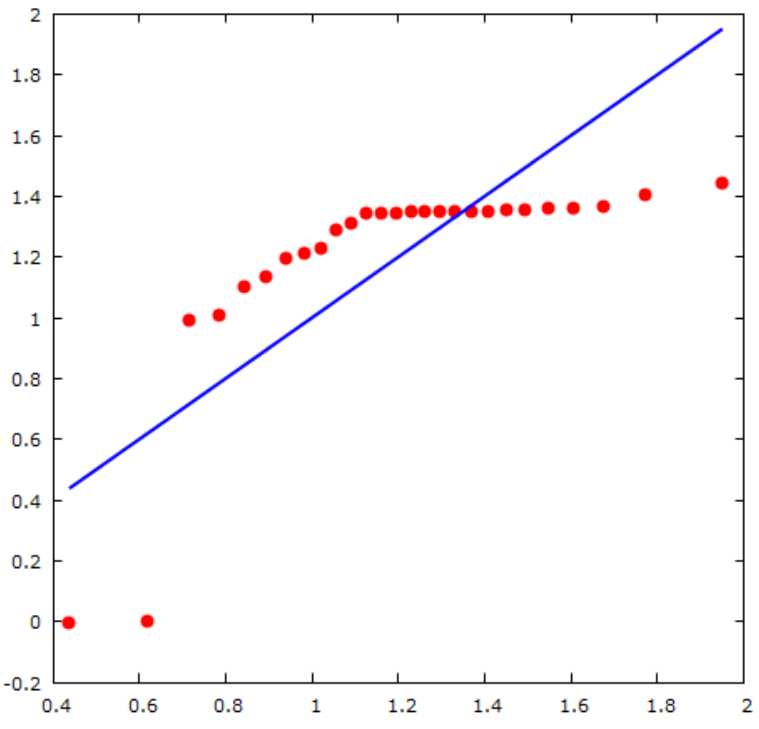

Fig. 8. Plot for throughput of Outdoor Location for Normality Check using LNSM

TABLE III

PATH LOSS EXPONENT FOR DIFFERENT ENVIRONMENTS

\begin{tabular}{cc}
\hline \hline Parameter & Values in outdoor location \\
\hline Mean & -59.192 \\
Median & -58.990 \\
Minimum & -63.052 \\
Maximum & -56.754 \\
Standard deviation & 1.0183 \\
C.V. & 0.017203 \\
Skewness & -0.87642 \\
Ex. Kurtosis & 2.1473
\end{tabular}

pursuit node and in terms of throughput the normality has been shown in Fig.10. The plots show that the nodes are performed well in outdoor locations as compared to the LNSM technique.

The pursuit node is programmed using Hybrid TLBO unilateral technique to reach to the anchor node. Fig. 11, Fig. 12 \& Fig.13 shows the searching pattern of pursuit node while searching for anchor node in outdoor location. The paths followed are simulated in SCILAB virtual environment. The results listed below is expressed in a format of all the RSSI values in a test field in upper half and lower half shows the path followed by pursuit node. As seen in the Fig.11 the pursuit node get the first RSSI value of $-117 \mathrm{dBm}$. The pursuit node after getting the first trails received the $-121 \mathrm{dBm}$, $-109 \mathrm{dBm},-112 \mathrm{dBm},-111 \mathrm{dBm},-120 \mathrm{dBm}$ RSSI values from nearby location. According to the algorithm the pursuit node moves to the new location of $-109 \mathrm{dBm}$ because $-109 \mathrm{dBm}$ is the largest value among all other values. Now the pursuit node learns to move to the largest value. This is the way teacher gets the best learner that is moving towards the largest value. Now Similarly $-109 \mathrm{dBm}$ gets eight new RSSI values from the nearby location but it will choose a new location of $-107 \mathrm{dBm}$ and the process continues till the pursuit node received $-40 \mathrm{dBm}$ value. In Fig.12, the pursuit node received the $-121 \mathrm{dBm}$ RSSI value and subjected to move to new location of $-105 \mathrm{dBm}$ and node keep on following the same rule 


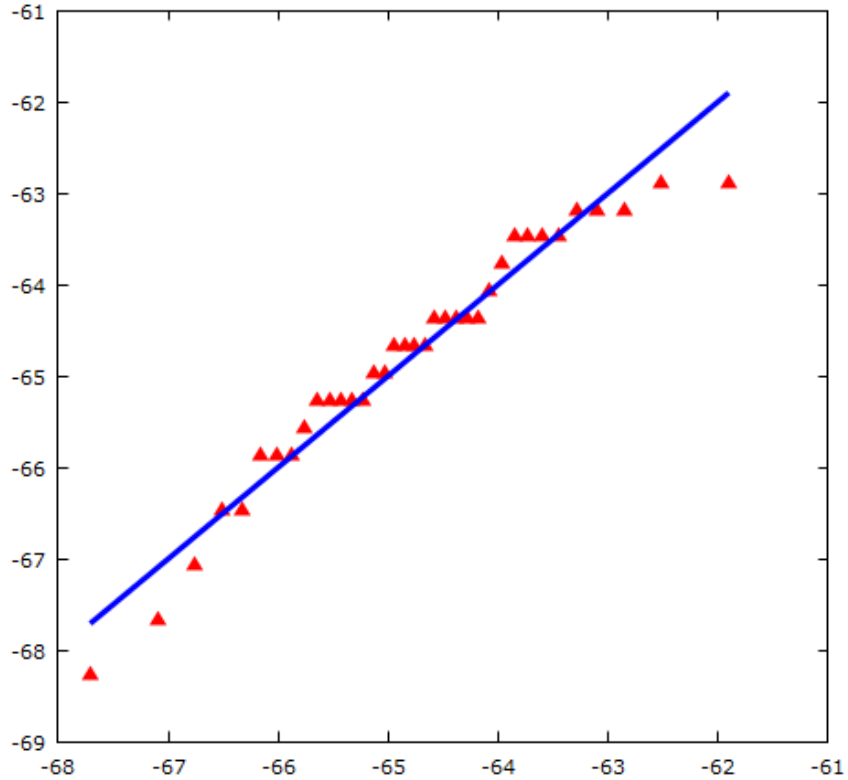

Fig. 9. Plot for Outdoor Location for Normality Check-Hybrid TLBO Unilateral Algorithm

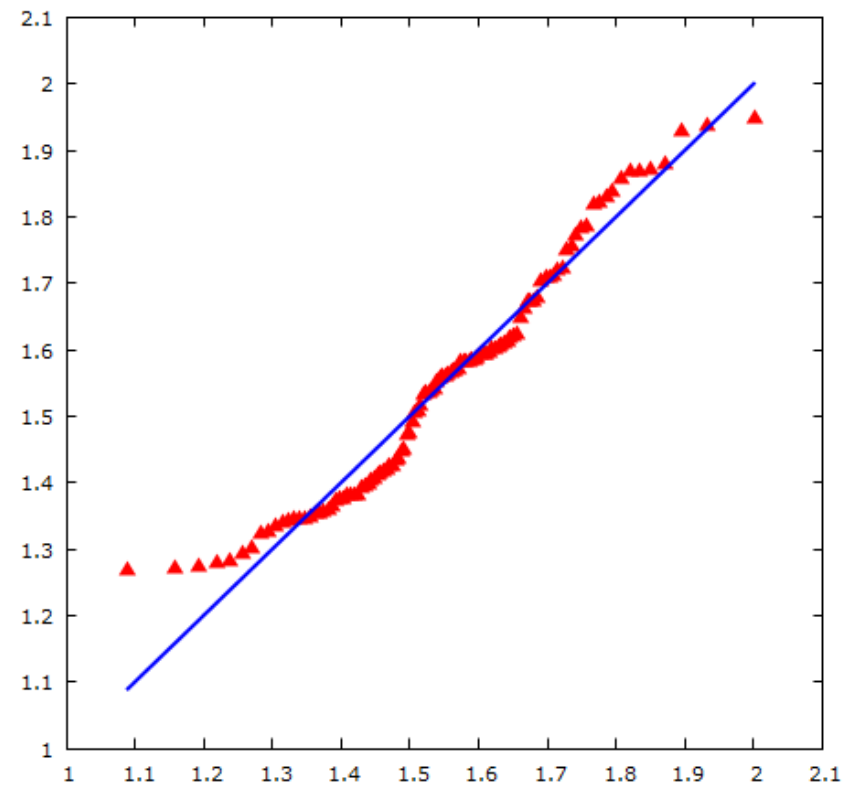

Fig. 10. Plot for throughput of Outdoor Location for Normality Check Hybrid TLBO Unilateral Algorithm

and reaches to the anchor node where it will receive $-40 \mathrm{dBm}$ RSSI value. Same is the case of Fig. 13 for dense forest outdoor location. In Fig.12 \& Fig.13 there is a deviation in path but finally reaches to the anchor node. As the RSSI values are faded and follow multipath in real environment so the path become deviated but the pursuit node is trained in a way to reach to the anchor node in any situation. This will make the anchor node fully discoverable in any outdoor location.

\section{CONCLUSION}

Two approaches of localization have been studied in this paper. The methods are aimed to localize the anchor node by

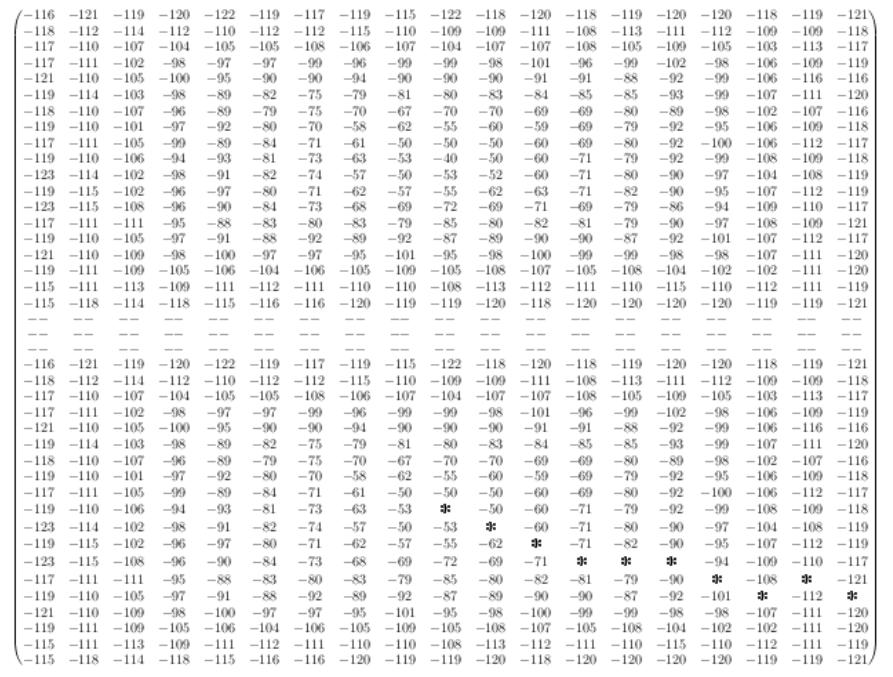

Fig. 11. Path as Computed by Hybrid TLBO -Unilateral Algorithm for Outdoor Location

pursuit node. The first method is traditional LNSM technique and second method is hybrid TLBO- unilateral technique. In LNSM technique the wireless channel is modeled. After modeling, the new equations of finding estimated distance have been derived for outdoor locations. The nodes proved to be not fully normally distributed using Shapiro Wilk and Jarque Bera tests. In the other method Hybrid TLBO- unilateral technique the pursuit node is trained as teacher and learner to follow the path of greater RSSI value than the previous RSSI value received from the anchor node. The tests have been conducted for outdoor location and found that the anchor node is fully discoverable by pursuit node. The nodes proved to be normally distributed using Shapiro Wilk and Jarque Bera tests. The standard deviation also found to be in range for outdoor locations. The Hybrid TLBO- unilateral is a flexible and easy to use algorithm that can be applied to any mobile node or fixed node. The localization problem in WSN can be deal with hybrid TLBO- unilateral Algorithm.

\begin{tabular}{|c|c|c|c|c|c|c|c|c|c|c|c|c|c|c|c|c|c|c|}
\hline & & & & & & & & & & & & & & & & & & \\
\hline & & & & & & & & & & & 21 & & it & -1200 & 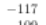 & 198 & & \\
\hline & 109 & $\begin{array}{l}-111 \\
-111\end{array}$ & $\begin{array}{l}-108 \\
-106\end{array}$ & $\begin{array}{l}-112 \\
-102\end{array}$ & -105 & $\begin{array}{l}-105 \\
-106\end{array}$ & $\begin{array}{l}-110 \\
-10,\end{array}$ & $\begin{array}{l}-100 \\
-106\end{array}$ & $\begin{array}{l}-1111 \\
-104\end{array}$ & $\begin{array}{l}-108 \\
-104 \\
-10\end{array}$ & $\begin{array}{l}-113 \\
-102\end{array}$ & $\begin{array}{l}1100 \\
104\end{array}$ & $\begin{array}{l}-111 \\
-104\end{array}$ & $\begin{array}{l}-111 \\
-107\end{array}$ & $\begin{array}{l}-109 \\
-108\end{array}$ & $\begin{array}{l}08 \\
{ }_{n}^{2}\end{array}$ & $\begin{array}{l}-1144 \\
-111\end{array}$ & \\
\hline & -112 & -105 & -101 & -98 & -1 & -97 & -99 & -99 & -100 & 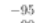 & , & 01 & -99 & -98 & 18 & -107 & -109 & -11 \\
\hline & & -110 & & & & & & & & & & & & & & & & \\
\hline & & & & -8 & & & & & & & & & & & & & $5_{15}^{5}$ & \\
\hline & $z$ & 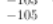 & 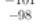 & $\begin{array}{l}-86 \\
-86\end{array}$ & & 68 & $\begin{array}{l}-10 \\
-62\end{array}$ & - & $\begin{array}{l}-19 \\
-57\end{array}$ & $\begin{array}{l}-69 \\
-5,5\end{array}$ & $\begin{array}{l}-11 \\
-64\end{array}$ & $\begin{array}{l}-11 \\
-\pi 2\end{array}$ & -8 & 89 & $\begin{array}{l}-39 \\
-98\end{array}$ & 5 & $\begin{array}{l}-115 \\
-113\end{array}$ & \\
\hline & 1 & -105 & -10 & -92 & -7 & 72 & -60 & & & -5 & & -66 & -81 & 77 & & & & \\
\hline & & & - & -91 & & & -6 & & & & & & & -94 & & & & -11 \\
\hline & & & & $\begin{array}{l}-91 \\
-9 x\end{array}$ & & & & & & & & & & 90 & & & & -11 \\
\hline & & & $\begin{array}{l}-1 \\
-1\end{array}$ & $\begin{array}{l}-9 \\
-9\end{array}$ & 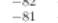 & $\begin{array}{l}71 \\
72\end{array}$ & & & & & & & & & & & & 11 \\
\hline & & & & & & & & & & & & & & & & & & \\
\hline & & & & & & & & & & & & & & & & & & \\
\hline & & $\begin{array}{l}-107 \\
-105\end{array}$ & $\begin{array}{l}-95 \\
-103\end{array}$ & & & 998 & -93 & & & $\begin{array}{c}-1 \\
-1\end{array}$ & & $\begin{array}{l}-97 \\
-105\end{array}$ & & & & & & -11 \\
\hline & & 14 & & & & 07 & & & & -11 & & & & & & & & \\
\hline & 6 & -117 & 19 & -121 & -120 & 20 & 20 & & & -118 & 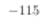 & -118 & 6 & -11 & 20 & 17 & -1 & \\
\hline & -- & - & - & -- & -- & -- & -- & - & -- & - & - & - & -- & -- & - & -- & & \\
\hline & & & & & & & & & & & & & & & & & & \\
\hline & & & & & & & & & & & & & & & & & & \\
\hline & & & & & & & & & & & & & & & & & & \\
\hline & & & & & & & & & & & & & & & & & & \\
\hline & & & & & & & & & & & & & & & & & & \\
\hline & & & & 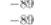 & -8 & " & -8 & & & & & & & $\begin{array}{l}-9 \\
-9\end{array}$ & & & & \\
\hline & & & & & & & & & & & & & & & & & & \\
\hline & & & & & & & & & & $\begin{array}{l}-4 \\
-5\end{array}$ & & & & & & & & \\
\hline & & & & & & & & & & & & & & & & & & \\
\hline & & & & & & & & & & & & & & & & & & \\
\hline & & & & & & & & & & & & & & & & & & \\
\hline & & & & & & & & & & & & & & & & & & \\
\hline & & & & & & & & & & & & & & & & & & \\
\hline & & & & & & & & & & & & & & & & & & \\
\hline & & -16 & & & & & & & & & & & & & & & & \\
\hline & & & & & & & & & & & & & & & & & & \\
\hline & & & & & & & & & & & & & & & & & & \\
\hline
\end{tabular}

Fig. 12. Path as Computed by Hybrid TLBO-Unilateral Algorithm for Indoor Location 


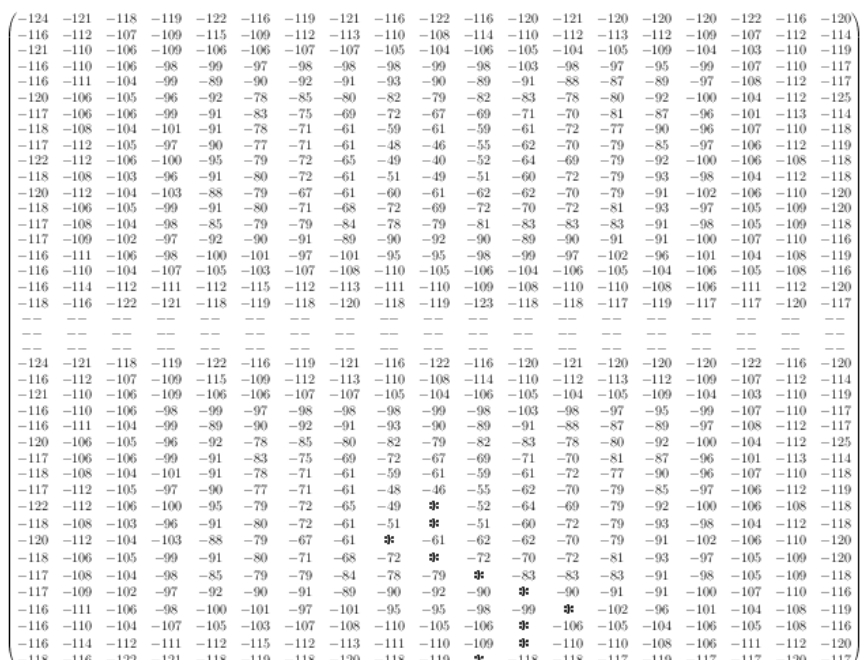

Fig. 13. Path as Computed by Hybrid TLBO-Unilateral Algorithm for Dense Forest Outdoor Location

\section{REFERENCES}

[1] Guerriero, M., et al., Some aspects of DOA estimation using a network of blind sensors. Signal Processing, 2008. 88(11): p. 2640-2650.

[2] Singh, S.P. and S. Sharma, Range free localization techniques in wireless sensor networks: A review. Procedia Computer Science, 2015. 57: p. 7-16

[3] Doherty, L. and L. El Ghaoui. Convex position estimation in wireless sensor networks. in INFOCOM 2001. Twentieth Annual Joint Conference of the IEEE Computer and Communications Societies. Proceedings. IEEE. 2001. IEEE.

[4] Bulusu, N., J. Heidemann, and D. Estrin, GPS-less low-cost outdoor localization for very small devices. IEEE personal communications, 2000. 7(5): p. 28-34.

[5] He, T., et al. Range-free localization schemes for large scale sensor networks. in Proceedings of the 9th annual international conference on Mobile computing and networking. 2003. ACM.

[6] Almuzaini, K.K. and T.A. Gulliver. A new distributed range-free localization algorithm for wireless networks. in Vehicular Technology Conference Fall (VTC 2009-Fall), 2009 IEEE 70th. 2009. IEEE.

[7] Aiello, M., R. de Jong, and J. de Nes. Bluetooth broadcasting: How far can we go? An experimental study. in Pervasive Computing (JCPC), 2009 Joint Conferences on. 2009. IEEE.

[8] Specification, Z., ZigBee Alliance. ZigBee Document 053474r06, Version, 2006. 1

[9] Chruszczyk, . and A. Zajc, Comparison of indoor/outdoor, RSSI-based positioning using 433, 868 or $2400 \mathrm{MHz}$ ISM bands. International Journal of Electronics and Telecommunications, 2016. 62(4): p. 395-399.

[10] Rao, R.V., Teaching-Learning-Based Optimization Algorithm, in Teaching Learning Based Optimization Algorithm2016, Springer. p. 9-39.

[11] Vivek Kaundal, Paawan Sharma, Devender Saini, Manish Prateek, Location Fingerprinting Supported Unilateral Algorithm based on Experimental Study of Localization in Disaster Prone Area International Journal of Computer Science and Information Security, 2016. 14: p. 162-175.
[12] Halder, S. and A. Ghosal, A survey on mobility-assisted localization techniques in wireless sensor networks. Journal of Network and Computer Applications, 2016. 60: p. 82-94.

[13] He, T., et al., Range-free localization and its impact on large scale sensor networks. ACM Transactions on Embedded Computing Systems (TECS), 2005. 4(4): p. 877-906.

[14] Anzai, D. and S. Hara An RSSI-based MAP localization method with channel parameters estimation in wireless sensor networks. in Vehicular Technology Conference, 2009. VTC Spring 2009. IEEE 69th. 2009. IEEE

[15] Cheng, G., Accurate TOA-based UWB localization system in coal mine based on WSN. Physics Procedia, 2012. 24: p. 534-540.

[16] Dakkak, M., et al., Indoor localization method based on RTT and AOA using coordinates clustering. Computer networks, 2011. 55(8): p. 17941803 .

[17] Gezici, S., A survey on wireless position estimation. Wireless personal communications, 2008. 44(3): p. 263-282

[18] Gharghan, S.K., et al., Accurate Wireless Sensor Localization Technique Based on Hybrid PSO-ANN Algorithm for Indoor and Outdoor Track Cycling. IEEE Sensors Journal, 2016. 16(2): p. 529-541.

[19] Pires, R.P., et al., Evaluation of an rssi-based location algorithm for wireless sensor networks. IEEE Latin America Transactions, 2011. 9(1): p. $830-835$

[20] Blywis, B., et al. A localization framework for wireless mesh networksanchor-free distributed localization in the des-testbed. in Indoor Positioning and Indoor Navigation (IPIN), 2010 International Conference on 2010. IEEE.

[21] Huang, C.-N. and C.-T. Chan, ZigBee-based indoor location system by k-nearest neighbor algorithm with weighted RSSI. Procedia Computer Science, 2011. 5: p. 58-65.

[22] Liu, W., et al. Radio map position inference algorithm for indoor positioning systems. in 2012 18th IEEE International Conference on Networks (ICON). 2012. IEEE.

[23] Luo, X., W.J. OBrien, and C.L. Julien, Comparative evaluation of Received Signal-Strength Index (RSSI) based indoor localization techniques for construction jobsites. Advanced Engineering Informatics, 2011. 25(2) p. $355-363$.

[24] Mao, G., B. Fidan, and B.D. Anderson, Wireless sensor network localization techniques. Computer networks, 2007. 51(10): p. 2529-2553.

[25] Meng, W., L Xie, and W. Xiao, Decentralized TDOA sensor pairing in multihop wireless sensor networks. IEEE Signal Processing Letters, 2013. 20(2): p. 181-184.

[26] Mesmoudi, A., M. Feham, and N. Labraoui, Wireless sensor networks localization algorithms: a comprehensive survey. arXiv preprint arXiv:1312.4082, 2013.

[27] Nasipuri, A. and K. Li. A directionality based location discovery scheme for wireless sensor networks. in Proceedings of the 1st ACM international workshop on Wireless sensor networks and applications. 2002. ACM

[28] Savvides, A., C.-C. Han, and M.B. Strivastava. Dynamic fine-grained localization in ad-hoc networks of sensors. in Proceedings of the 7th annual international conference on Mobile computing and networking. 2001. ACM.

[29] Hanusz, Z., J. Tarasinska, and W. Zielinski, Shapiro-Wilk test with known mean. REVSTAT-Statistical Journal, 2016. 14(1): p. 89-100.

[30] Thadewald, T. and H. Bning, JarqueBera test and its competitors for testing normalitya power comparison. Journal of Applied Statistics, 2007. 34(1): p. $87-105$. 